\title{
IKZF1 Protein Variant
}

National Cancer Institute

\section{Source}

National Cancer Institute. IKZF1 Protein Variant. NCI Thesaurus. Code C158145.

A variation in the amino acid sequence for DNA-binding protein Ikaros. 